Rev. Ter. Ocup. Univ. São Paulo, v. 22, n. 2, p. 145-152, maio/ago. 2011.

\title{
Abordagem funcional e centrada no cliente na reabilitação de idoso com demência de Alzheimer avançada - relato de caso*
}

\section{Functional approach and centered-client pratic in rehabilitation of severe Alzheimer disease's older adult - case report}

\author{
Fabiana Carla Matos da Cunha ${ }^{1}$, Luciana Cristina Matos da \\ Cunha ${ }^{2}$, Hercília Martins da Silva ${ }^{3}$, Érica de Araújo \\ Brandão Couto ${ }^{4}$
}

\begin{abstract}
CUNHA, F. C. M., CUNHA, L. C. M., SILVA, H. M., COUTO, E. A. B. Abordagem funcional e centrada no cliente na reabilitação de idoso com demência de alzheimer avançada - relato de caso. Rev. Ter. Ocup. Univ. São Paulo, v. 22, n. 2, p. 145-152, maio/ago. 2011.

RESUMO: A Doença de Alzheimer (DA) é uma doença cerebral degenerativa que compromete a cognição e a realização das atividades de vida diária (AVDs). Os tratamentos disponíveis apenas minimizam os déficits cognitivos e as alterações de comportamento, sendo necessárias medidas que melhorem a qualidade de vida de pacientes e cuidadores. Objetivos: Apresentar os resultados da intervenção domiciliária de TO usando abordagem funcional e centrada no cliente associada ao tratamento farmacológico em idoso com DA avançada. Descrição do caso clínico: Paciente de 64 anos, sexo masculino, previamente hígido, que, em 2004, apresentou piora progressiva das funções cognitivas, sendo diagnosticado com provável DA. Em 2006, foi avaliado pelo serviço de Terapia Ocupacional (TO) do Hospital Socor/BH, apresentando baixos escores nos testes cognitivos e funcionais (Mini-Mental; Testes de Fluência Verbal e do Relógio; Índice de Katz; Medida Canadense do Desempenho Ocupacional - COPM e Time diary). Observou-se dependência total em AVDs, grande insatisfação da cuidadora relacionados às alterações comportamentais apresentadas pelo
\end{abstract}

* Relato de caso de um idoso atendido pela equipe, em domicílio. Está sendo encaminhado pela primeira vez para publicação na Revista de Terapia Ocupacional da Universidade de São Paulo, sendo apresentado, anteriormente, como Poster no Congresso Mineiro de Geriatria e Gerontologia, em Ouro Preto - outubro/2007 e no Congresso Brasileiro de Geriatria e Gerontologia, em Porto Alegre - setembro/2008.

1. Terapeuta Ocupacional especialista em Gerontologia pela Universidade Federal de Minas Gerais; Mestranda em Neurociências/UFMG; Referência Técnica do serviço de saúde do idoso do Hospital Municipal São Judas Tadeu - Ribeirão das Neves/MG.

Marco Túlio Gualberto Cintra - Médico Geriatra do Núcleo de Geriatria e Gerontologia do Hospital das Clínicas da Universidade Federal de Minas Gerais; Mestrando em Saúde do Adulto / UFMG.

2. Fonoaudióloga; Mestre em ciências da saúde: infectologia e medicina tropical da Universidade Federal de Minas Gerais (UFMG); Supervisora Clínica Fonoaudiologia Gerontológica da Universidade Federal de Minas Gerais (UFMG).

3. Terapeuta Ocupacional Mestre em Ciências da Reabilitação pela Universidade Federal de Minas Gerais; Coordenadora do Serviço de Terapia Ocupacional do Hospital Socor.

4. Fonoaudióloga, Especialista em Gerontologia pela Sociedade Brasileira de Geriatria e Gerontologia, Professora Assistente do Curso de Fonoaudiologia da Universidade Federal de Minas Gerais (UFMG); Coordenadora do Serviço de Fonoaudiologia do Centro de Referencia do Idoso do Hospital das Clínicas da Universidade Federal de Minas Gerais (UFMG).

Endereço para correspondência: Érica de Araújo Brandão Couto - Departamento de Fonoaudiologia - Faculdade de Medicina, Universidade Federal de Minas Gerais - Av: Alfredo Balena, 190, Santa Efigênia, Belo Horizonte, MG. CEP 30130-100. ecoutobhz@gmail.com 
paciente (alucinação, perambulação, agitação e inversão do ciclo sono-vigília), interação social reduzida e dependência crescente em AVDs básicas, além da desorganização da rotina ocupacional. Foram realizadas três sessões semanais no domicílio, com duração de 1 hora e acompanhamento pela neurologia e clínica médica. Observou-se melhora nos testes após três meses de tratamento, sustentada por 8 meses, contrapondo-se a evolução descrita na literatura sobre a progressão inexorável da incapacidade funcional. O Índice de Katz, ao contrário da COPM e time diary, não descreveu a melhora qualitativa observada no desempenho das AVDs. Conclusão: A intervenção domiciliária de TO associada ao tratamento medicamentoso mostrou efeitos significativos no caso apresentado.

DESCRITORES: Doença de Alzheimer; Terapia ocupacional; Reabilitação; Terapia não-dirigida; Resultado de tratamento.

\section{INTRODUÇÃO}

A doença de Alzheimer (DA) é considerada a causa mais freqüente de transtornos demenciais no idoso, afetando pelo menos $5 \%$ dos indivíduos com mais de 65 anos e $20 \%$ daqueles com mais de 80 anos. O impacto importante da doença na vida dos pacientes e de seus familiares é acrescido de enorme custo financeiro para a sociedade. Estima-se que os gastos anuais com DA nos EUA é de aproximadamente 30 bilhões, além de custos indiretos relacionados ao impacto na saúde e tempo despendido pelos cuidadores (HERRERA et al., 1998; MACHADO, 2006).

A DA é uma doença cerebral degenerativa caracterizada por perda progressiva da memória e outras funções cognitivas superiores, que prejudicam o paciente em suas atividades de vida diária (AVDs), podendo ocorrer, também, distúrbios de comportamento e sintomas neuropsiquiátricos (BPSD) (MACHADO, 2006; NITRINI et al., 2005). As AVDs podem ser classificadas em básicas, instrumentais e avançadas. As básicas relacionam-se as atividades relacionadas aos cuidados pessoais e necessárias a automanutenção do indivíduo, como alimentação, vestuário, transferência, continência e banhar-se . As instrumentais seriam atividades mais complexas, necessárias para a vida independente no lar e na comunidade, como cuidados com a casa, preparo de refeições, etc. Em contrapartida as AVDs avançadas referem-se a atividades sociais, de trabalho e lazer do indivíduo (PFEFFER et al., 1982).

Sabe-se que o nível de incapacidade funcional do sujeito pode ser avaliado por meio das AVDs, sendo atualmente este um dos critérios utilizados para classificação das fases da demência. O grau de comprometimento também é avaliado com base no nível de prejuízo cognitivo. $\mathrm{Na}$ fase inicial observa-se comprometimento da memória, das atividades sociais e desorientação progressiva em relação ao tempo e espaço com repercussões em atividades instrumentais complexas. Com a evolução para a fase intermediária, verifica-se freqüentemente deterioração mais acentuada da memória, desenvolvimento de afasia, apraxia, agnosia e alterações visoespaciais, sendo necessária maior assistência na realização das AVDs básicas e instrumentais. Quando todas as funções cognitivas encontram-se gravemente comprometidas, com o paciente acamado necessitando de assistência integral, fala-se em fase avançada da doença (NITRINI et al., 2005).

Apesar dos esforços freqüentes de pesquisas, até o presente momento não fora documentado tratamento eficaz capaz de curar ou reverter à deterioração provocada pela DA. Os tratamentos disponíveis visam aliviar os déficits cognitivos, alterações de comportamento e melhorar a qualidade de vida dos pacientes e familiares através do uso associado de medicamentos e da abordagem nãofarmacológica e multidisciplinar (ENGELHARDT et al., 2005).

Autores têm apontado melhora no desempenho cognitivo e funcional de pacientes em fases iniciais da DA com a utilização de intervenções psicossociais e técnicas de reabilitação cognitiva, principalmente quando associado ao tratamento farmacológico preconizado. Contudo, poucos estudos se propuseram a elucidar a eficácia do tratamento não medicamentoso nas AVD's de sujeitos em fases mais evoluídas da DA, sendo encontradas diversas controversas quanto a sua validade (NITRINI, 2005; 2006; ABRISQUETA-GOMEZ et al., 2004; ÁVILA et al., 2004; ÁVILA, 2003; BOTTINO et al., 2002).

O objetivo do presente estudo foi descrever os resultados da intervenção domiciliária de terapia ocupacional (TO) utilizando abordagem funcional e centrada no cliente, associado ao tratamento medicamentoso, em um idoso com diagnóstico de provável DA em fase avançada. 


\section{Descrição do Caso Clínico}

H.M., 64 anos, sexo masculino, casado, técnico em contabilidade, residindo com familiares em Belo Horizonte. Não possui histórico pregresso de outras patologias, etilismo e tabagismo.

Em 2004, procurou atendimento médico devido à queixa de freqüentes esquecimentos. Em 2005, após avaliação clínica, funcional e neuropsicológica especializada, estabeleceu-se o diagnóstico de provável DA fase intermediária, sendo então, afastado do trabalho. Encontrava-se em tratamento com a clínica médica e neurologia, em uso de Donepezila, Mirtazapina, Risperidona e Diazepam desde 2005. O paciente, já com DA em fase avançada, foi encaminhado ao serviço domiciliário de Terapia Ocupacional (TO) do Hospital Socor/Belo Horizonte, em outubro de 2006, e submetido novamente à avaliação cognitiva e funcional. As avaliações foram realizadas por meio da observação direta do paciente no ambiente domiciliar, entrevista com sua esposa - cuidadora principal - e aplicação de testes padronizados a saber: Mini Exame do Estado Mental (MEEM), Teste de Fluência Verbal - categoria animais e frutas, Teste do Relógio, Índice de Katz e Medida Canadense do Desempenho Ocupacional (COPM). Os dois últimos foram direcionados a cuidadora (LAW et al., 2009; NITRINI et al., 2005).

O COPM é um instrumento padronizado, em formato de entrevista semi-estruturada que possibilita identificar e priorizar questões do dia-a-dia que interferem no desempenho ocupacional. Desempenho ocupacional, é definido, em termos gerais, como a participação do indivíduo nas diversas atividades de vida diária (LAW et al., 2009).

Essa medida baseia-se no modelo da prática centrada no cliente, uma vez que incorpora o ambiente e as expectativas individuais, assegurando que as questões levantadas sejam de relevância para o cliente, que é encorajado a identificar até cinco atividades que ele considere como as de mais difícil execução nas áreas de auto-cuidado, produtividade e lazer e, depois, pontuá-las, dando-lhes uma nota de zero a dez em relação a seu desempenho e satisfação. Pode ser aplicado junto ao cliente ou terceiros (LAW et al., 2009).

Realizou-se, também, a avaliação qualitativa do desempenho ocupacional, operacionalizada através da análise e descrição do tempo gasto pelo paciente na realização de suas ocupações diárias (AVDs básicas e instrumentais, atividades sociais/lazer, sono e trabalho), pelo Time Diary ou relógio de atividades (DOIMO, 2006). As informações foram relatadas pela cuidadora descrevendo três momentos da rotina ocupacional do idoso: antes do diagnóstico de DA, no momento da primeira avaliação e 8 meses após as intervenções.

Ao contrário dos testes cognitivos, avaliação funcional permitiu registrar o impacto da DA sobre o desempenho das atividades cotidianas do idoso.

A análise dos dados coletados à admissão demonstrou prejuízo cognitivo e funcional significativos, com baixos escores em todos os testes, como descrito na Tabela 1 e Gráfico 1.

Tabela 1. Resultado dos testes aplicados no momento da avaliação e oito meses após a intervenção

\begin{tabular}{c|c|c}
\hline & $\mathbf{2 4 / 0 8 / 2 0 0 6}$ & $\mathbf{2 7 / 0 4 / 2 0 0 7}$ \\
\hline MEEM & $7 / 30$ & $9 / 30$ \\
\hline Índice de Katz & Dependência total & Dependência total \\
\hline Teste de Fluência Verbal & $\begin{array}{c}\text { Animais }-1 \\
\text { Frutas }-0\end{array}$ & $\begin{array}{c}\text { Animais }-3 \\
\text { Frutas }-3\end{array}$ \\
\hline Teste do Relógio & 2 & 3 \\
\hline COPM & $\begin{array}{c}\text { Desempenho }-3,7 \\
\text { Satisfação }-2,0\end{array}$ & $\begin{array}{c}\text { Desempenho }-6 \\
\text { Satisfação }-6,3\end{array}$ \\
\hline
\end{tabular}

O idoso apresentou desempenho insatisfatório nos itens do MEEM relacionados à orientação temporal e espacial, nomeação, evocação, cálculo e escrita. A fluência verbal encontrava-se reduzida. O Teste do Relógio indicou alterações visoespaciais graves. O Índice de Katz demonstrou dependência total nas AVDs. A COPM revelou grande insatisfação e sobrecarga da cuidadora, relacionados às alterações comportamentais apresentadas pelo paciente (alucinação, perambulação, agitação e inversão do ciclo sono-vigília), interação social reduzida e dependência crescente em AVDs básicas, com necessidade de auxílio total para alimentação, banho e vestuário. 
Conforme o Time Diary, no momento da avaliação, a rotina ocupacional do paciente encontrava-se completamente alterada e desorganizada, com abandono de atividades significativas, como trabalho e lazer e grande tempo despendido para o sono e AVDs básicas.

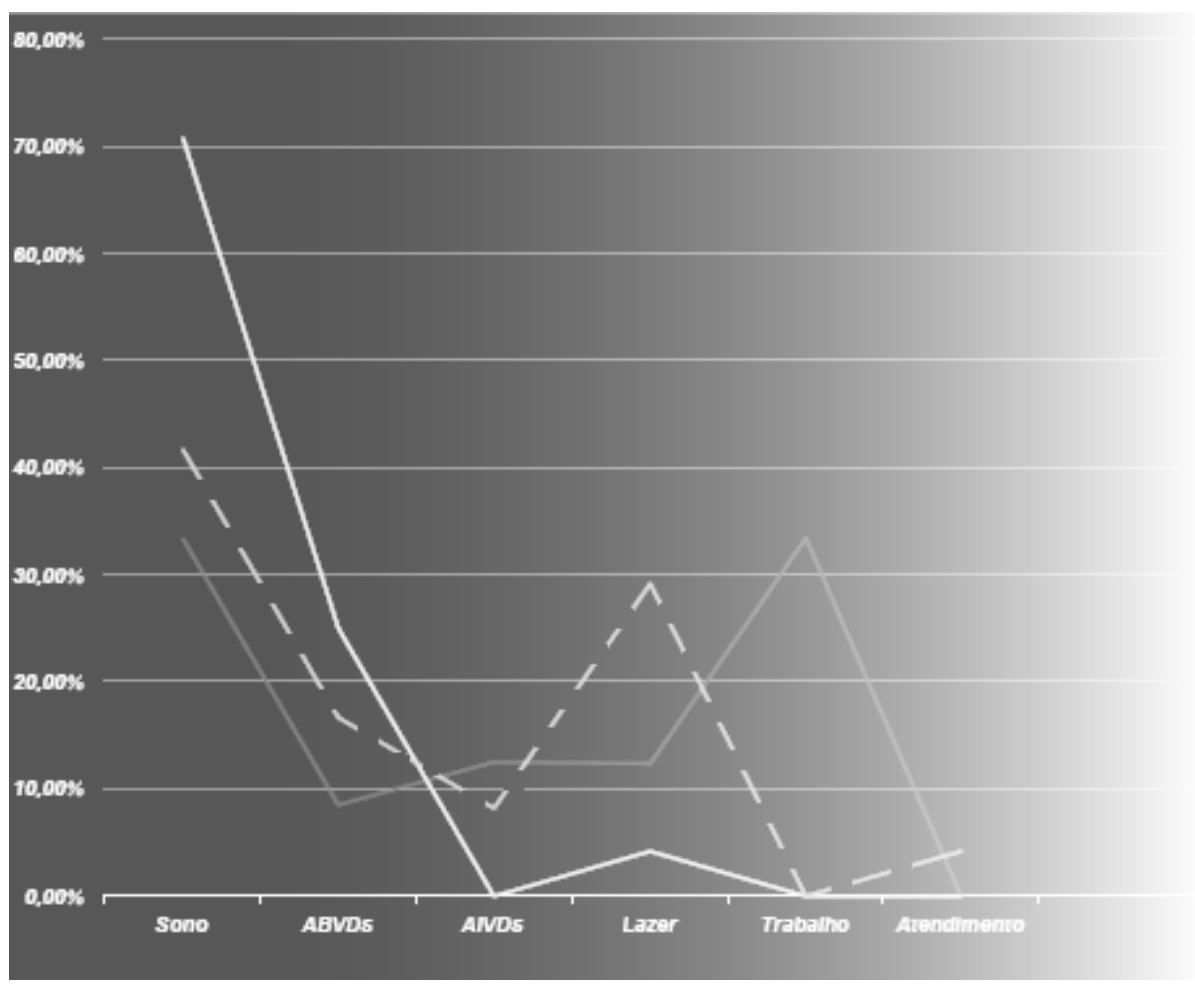

Rotina Ocupacional prévia__ Rotina Ocupacional na $1^{\mathrm{a}}$ avaliação Rotina Ocupacional após intervenção ABVDs: Atividades Básicas de Vida Diária; AIVDs: Atividades Instrumentais de Vida Diári

Gráfico 1. Distribuição das ocupações diárias do idoso (time diary) antes do diagnóstico, no momento da avaliação e oito meses após a intervenção da terapia ocupacional

Com base nos resultados expostos, planejou-se uma intervenção com o objetivo de minimizar as limitações nas AVDs, estimular as habilidades cognitivas remanescentes e controlar as alterações comportamentais e psicológicas da demência (BPSD). Este plano considerou os problemas apontados como causadores de insatisfação e sobrecarga da cuidadora, obtidos na COPM.

A intervenção terapêutica ocupacional consistiu em atendimentos individuais no domicílio, três vezes por semana, com duração de 60 minutos, durante o período de oito meses. Utilizou-se a abordagem funcional e centrada no cliente, descrita anteriormente, associada às estratégias de adaptação do ambiente, treinamento de AVDs e reabilitação cognitiva ( terapia de orientação para a realidade, terapia de reminiscências, pareamento de estímulos e aprendizagem sem erro).

A terapia de orientação para realidade (TOR) tem como princípio apresentar de forma organizada e contínua os dados da realidade e do contexto ao paciente, criando estímulos ambientais que facilitem a orientação temporal e espacial. Na TOR tenta-se engajar o indivíduo em interações sociais e melhorar a comunicação por meio de informação contínua, sinalizações no ambiente, linguagem verbal clara ou não verbal, através atividades (lúdicas ou não-lúdicas) adequadas às dificuldades apresentadas. A terapia de reminiscência, que é uma variação da TOR, tem como objetivo estimular o resgate de informações por meio de figuras, fotos, músicas, jogos e outros estímulos relacionados à juventude dos pacientes. Essa técnica tem sido muito utilizada para resgatar emoções vividas previamente, gerando maior socialização e entretenimento como parte da terapia. O pareamento de estímulos utiliza-se de informações visuais e verbais para facilitar a evocação do nome de pessoas com base em associações 
CUNHA, F. C. M. et al. Abordagem funcional e centrada. Rev. Ter. Ocup. Univ. São Paulo, v. 22, n. 2, p. 145-152, maio/ago. 2011.

de características físicas. Baseia-se no princípio de que o envolvimento de múltiplos sistemas sensoriais na aquisição da informação associa-se ao aumento da evocação tardia. A técnica "aprendizagem sem erros" reduz as chances de erros do paciente, propiciando seu sucesso na execução da tarefa. Baseia-se no princípio de que a aprendizagem de um procedimento fortalece a emissão de uma resposta, dentre uma gama de várias respostas possíveis. Se a resposta errada é repetida, acaba por fortalecer-se e dificultar a aprendizagem correta da tarefa. Dessa maneira, a possibilidade de obter sucesso aumenta a motivação do paciente e se associa à facilitação da aprendizagem e melhora a retenção de informações (FRASER, 1992).

Para conseguir reestruturar a rotina funcional do idoso e controlar as alterações de comportamento foi necessário, primeiramente, orientar e treinar a cuidadora quanto ao estabelecimento de atividades regulares e previsíveis para o idoso.

A realização de AVDs básicas (escovar os dentes, vestir roupas, tomar banho e alimentar-se) foi facilitada, através da organização prévia de materiais, instruções verbais e demonstrações. Para simplificar as atividades e reduzir o esforço cognitivo do paciente, tarefas específicas eram divididas em etapas e o paciente era encorajado pela TO a participar daquelas que conseguia. A esposa foi orientada a manter o treinamento além do período de atendimento, oferecendo assistência física, somente, quando as estratégias citadas falhassem.

O treinamento incluiu, também, o manejo nãofarmacológico dos Distúrbios de Comportamento e Sintomas Neuropsiquiátricos da Demência - BPSD. Atividades expressivas e de reminiscências, foram re-introduzidas, gradativamente, e permitiram maior interação social do paciente e a organização de sua rotina. Para o controle da perambulação e das alterações do sono, eram realizadas diariamente atividades físico-corporais leves (caminhadas e dança sênior). As alucinações e agitação foram abordadas através da retirada de estímulos ambientais distratores, como espelhos, barulho da TV ou excesso de utensílios durante as refeições ou outras tarefas (CARAMELLI; BOTTINO, 2007). Todas as atividades realizadas faziam parte do contexto do paciente, eram compatíveis com suas habilidades cognitivas e readaptadas a cada sessão.

A evolução do paciente durante o período de intervenção foi monitorada por meio de reavaliações trimestrais que consistiam na reaplicação dos testes utilizados à admissão. Durante todo este período houve aderência adequada ao tratamento medicamentoso e o paciente manteve-se em acompanhamento médico, sem hospitalizações.
Os resultados obtidos com a intervenção da TO foram significativos, sendo observado aumento sustentado do MEEM, teste do relógio e de fluência verbal (Tabela 1).

De acordo com o Índice de Katz, o paciente manteve dependência total em AVDs, apesar da melhora funcional visível observada. A COPM detectou indiretamente esta melhora, demonstrando aumento da satisfação da cuidadora em relação ao desempenho do paciente na alimentação, banho e vestuário .

O Time Diary, conforme demonstrado no Gráfico 1, também, indicou mudança no padrão das atividades realizadas pelo paciente ao longo do dia. Antes da manifestação dos sintomas da DA, o sono e o trabalho ocupavam aproximadamente $33 \%$ do tempo e o lazer e AVDs básicas e instrumentais $10 \%$ a $15 \%$, respectivamente. Quando foi admitido no serviço de TO, o sono e as AVDs básicas, auxiliadas pela cuidadora, ocupavam juntas $95 \%$ do tempo gasto diariamente. A atuação domiciliária da TO diminuiu o tempo destinado ao sono e AVDs básicas para 40 e 15\% respectivamente, distribuindo-o com atividades de lazer/ socialização (30\%) e AVDs instrumentais assistidas pela cuidadora $(10 \%)$.

\section{DISCUSSÃO}

No caso apresentado, a melhora funcional e cognitiva evidenciada nos testes contrapõe-se a evolução natural da doença descrita na literatura. O MEEM, teste de rastreio e acompanhamento do declínio cognitivo, apresentou alta sustentada de 2 pontos a partir do $3^{\circ}$ mês de intervenção. Esse resultado opõe-se ao decréscimo de 2 a 2,5 pontos/ano esperado na DA durante a vigência somente do tratamento farmacológico, indicando resultado positivo da intervenção de TO. O teste do relógio apresentou discreta melhora concordante com a literatura e, o Teste de Fluência Verbal evidenciou ganho importante, não encontrado nas análises de evolução (NITRINI, 2005; 2006; ABRISQUETAGOMEZ et al., 2004; ÁVILA et al., 2004; ÁVILA, 2003; BOTTINO et al., 2002).

Desde 2006, estudos têm demonstrado que o uso de donepezila pode melhorar a cognição e preservar a funcionalidade em pacientes com DA avançada, assim como apresentar ação benéfica nos sintomas comportamentais e na redução da sobrecarga do cuidador. A donepezila é o único anticolinesterásico indicado pelo Food and Drug Administration (FDA) para uso na DA avançada e o uso desta medicação para tal finalidade já fora liberado pela Agência Nacional de Vigilância Sanitária (WINBLAD 2006; HOMMA, 2009). No entanto, a donepezila age reduzindo 
CUNHA, F. C. M. et al. Abordagem funcional e centrada. Rev. Ter. Ocup. Univ. São Paulo, v. 22, n. 2, p. 145-152, maio/ago. 2011.

somente em parte o déficit colinérgico existente no cérebro do paciente com DA, exercendo assim os seus efeitos sintomáticos modestos de acordo com a literatura. Por isso, têm sido investigados intensamente novos tratamentos farmacológicos e não-farmacológicos, que possam obter maiores efeitos no tratamento da DA (HOGAN, 2008).

O paciente estudado já encontrava-se em uso desta medicação a 18 meses antes do início da intervenção da TO, não sendo, portanto, o responsável isolado pela melhora, haja vista que os efeitos da medicação ocorrem de três a quatro meses após seu uso em dose plena (HOGAN, 2008).

Estudos recentes descrevem que o programa de reabilitação cognitiva, associado ao tratamento medicamentoso, apresenta efeitos satisfatórios na melhora de testes cognitivos e AVDs após cinco meses a um ano do início da intervenção (BOTTINO, 2005; CARAMELLI, 2007). Todavia, os estudos não têm abordado especificamente a manutenção desses ganhos em relação à funcionalidade, demonstrando, apenas, que o treino da memória em pacientes com DA leve a moderada promove melhora da memória explícita que se estende para habilidades funcionais temporariamente. Somando a isso, trabalhos utilizando tratamento não-farmacológico multidisciplinar estruturado na DA avançada são muito escassos (HOGAN, 2008).

No caso apresentado, os ganhos funcionais foram verificados a partir do $3^{\circ}$ mês de atendimento e mantiveramse por mais cinco meses de acompanhamento. Resultado semelhante foi encontrado em um ensaio clinico randomizado que investigou a eficácia da terapia ocupacional domiciliária no funcionamento diário de pacientes com demência e de seus respectivos cuidadores (GRAFF et al., 2006). Os autores atribuíram tais resultados ao treinamento dos cuidadores para promover estimulação e supervisão ao familiar e à intervenção mais individualizada e centrada nas dificuldades e demandas apontadas por eles, no contexto domiciliar.

Logo, a melhora sustentada observada neste relato de caso pode ser justificada pelo treinamento de habilidades funcionais básicas e sociais remanescentes do idoso, no ambiente domiciliar com a participação de sua cuidadora, associada ao tratamento medicamentoso, conforme já evidenciado pela literatura (GRAFF et al., 2006).

É importante ressaltar, também, que as habilidades cognitivas residuais do paciente foram estimuladas de forma intensiva utilizando-se técnicas de reabilitação cognitiva a partir de atividades sociais e de lazer de seu contexto, simplificadas e adaptadas; e não apenas, por exercícios cognitivos descontextualizados, que poderiam se tornar cansativos e pouco prazerosos.

Estudos descrevem, também, que o treino de AVDs, pode produzir rápidas alterações na rotina ocupacional do sujeito, entretanto, os efeitos dessa intervenção são limitados à modificação / adaptação da tarefa e do ambiente e dependem da confiabilidade e consistência do prestador de atendimento para o sucesso. Tal procedimento favorece a automatização das tarefas funcionais e reduz a demanda cognitiva destas, não exigindo do paciente raciocínio e aprendizado. Há, contudo, necessidade constante de suporte, educação e treinamento do cuidador, ao invés de intervir apenas com o cliente (TOGLIA, 2002; BOTTINO, 2005).

O conhecimento exaustivo do desempenho ocupacional por meio da entrevista, COPM e time diary permitiu acompanhar a evolução do paciente nas AVDs, o que não seria possível somente com as avaliações cognitivas e o Índice de Katz. As intervenções proporcionaram, além da melhora nos testes cognitivos, a reintrodução de atividades que foram abandonadas devido à progressão dos déficits e a redistribuição gradual do tempo gasto nas AVDs. O COPM e o time diary foram os únicos testes capazes de detectar os benefícios obtidos. O índice de Katz, muito utilizado pela geriatria e gerontologia para classificar a condição funcional de idosos, não foi sensível o suficiente para detectar as alterações na rotina e desempenho ocupacional do paciente. Isto provavelmente ocorreu, porque trata-se de uma escala do tipo likert que classifica o estado funcional em apenas três níveis: independente, necessita de ajuda ou dependente, não sendo capaz de identificar estados intermediários (PFEFFER, 1982).

De acordo com Machado (2006), a interferência dos déficits cognitivos nas AVDs, atividades sociais e ocupacionais do paciente, podem servir não somente como um auxílio importante para o diagnóstico, mas também para estimar o impacto da doença sobre o indivíduo, além de facilitar a monitoração do plano de reabilitação.

Esse relato demonstrou que a intervenção da terapia ocupacional domiciliária utilizando a abordagem funcional e centrada no cliente, associada ao uso de Donepezila, promove efeitos satisfatórios em paciente com DA avançada, proporcionando impacto positivo sobre o cuidador, reduzindo a dependência funcional do paciente e promovendo a estabilização temporária da doença, apesar do caráter progressivo da mesma.

Por fim, novos estudos, com maior número de pacientes e tempo de acompanhamento, são necessários, para que se possa verificar a fidedignidade, duração e generalização desses benefícios. 
CUNHA, F. C. M. et al. Abordagem funcional e centrada. Rev. Ter. Ocup. Univ. São Paulo, v. 22, n. 2, p. 145-152, maio/ago. 2011.

CUNHA, F. C. M., CUNHA, L. C. M., SILVA, H. M., COUTO, E. A. B. Functional approach and centered-client pratic in rehabilitation of severe alzheimer disease's older adult - case report. Rev. Ter. Ocup. Univ. São Paulo, v. 22, n. 2, p. 145-152, maio/ago. 2011.

\begin{abstract}
Alzheimer's disease (AD) is a degenerative brain disease that affects cognition and perform activities of daily living (ADLs). The treatments available only minimize the cognitive deficits and behavioral changes, being necessary measures to improve the quality of life of patients and caregivers. Objectives: To present the results of interventions using Ocupational Therapy at home and functional approach focusing on customer conjunction with pharmacological treatment in elderly patients with advanced DA. Description of the case: A 64 year old male, previously healthy, who in 2004 showed progressive worsening of cognitive function, being diagnosed with probable AD. In 2006 it was assessed by the department of Occupational Therapy (OT), Hospital Socor-BH, showing low scores on cognitive tests and functional (Mini-Mental, Verbal Fluency Test and the Clock; Katz Index, the Canadian Occupational Performance Measure - Time diary and COPM). There is total dependence in ADL, caregiver's great dissatisfaction about the behavioral changes shown and the need for total aid to feed,bath and clothing, plus the disruption of routine occupational. They performed three sessions per week at home, lasting 1 hour and monitored by the neurology and internal medicine. An improvement in the tests after three months of treatment, sustained for 8 months, opposing the developments described in the literature about the inexorable progression of disability. The Katz Index, unlike the time and COPM diary, did not describe the observed qualitative improvement in the performance of ADLs. Conclusion: The home of OT intervention related to drug treatment showed significant effects in the case present.
\end{abstract}

KEY WORDS: Alzheimer disease; Occupational therapy; Rehabilitation; Nondirective therapy; Treatment outcome.

\title{
REFERÊNCIAS
}

1. ABRISQUETA-GOMEZ, J; et al. A longitudial study of a neuropsychological rehabilitation program in Alzheimer's disease. Arq. Neuro-Psiquiatr., v. 62, n. 3b, p.7 78-783, 2004.

2. ÁVILA, R. Resultados da reabilitação neuropsicológica em paciente com doença de Alzheimer leve. Rev. Psiquiatr. Clín. v. 30, n. 4, p. 139-146, 2003.

3. ÁVILA, R.; et al. Neuropsychological rehabilitation of memory deficits and activities of daily living in patients with Alzheimer's disease: a pilot study. Braz. J. Med. Biol. Res, v. 37, n. 11, p. 1721-1729, 2004.

4. BOTtiNO, C. M. C.; et al. Reabilitação cognitiva em pacientes com doença de Alzheimer: Relato de trabalho em equipe multidisciplinar. Arq. Neuro-Psiquiatr, v. 60, n. 1, p. 70-79, 2002.

5. BOTTINO, C. M. C. O tratamento de longo prazo está indicado para pacientes com doença de Alzheimer? Rev. Psiquiatr. Clín., v. 32, n. 6, p. 341-342, 2005.

6. CARAMELLI, P.; BOTTINO, C. M. C. Tratando os sintomas comportamentais e psicológicos da demência (SCPD). J. Bras. Psiquiatr., v. 56, n. 2, p. 83-87, 2007.
7. DOIMO, L. A.; DERNTL, A. M. Uso do tempo no cotidiano de idosos: um método indicador do estilo e modo de vida na velhice. Rev. Bras. Geriatr. Gerontol. v. 9 n. 1, 2006.

8. ENGELHARDT, E.; et al. Tratamento da doença de Alzheimer: recomendações e sugestões do Departamento Científico de Neurologia Cognitiva e do Envelhecimento da Academia Brasileira de Neurologia. Arq. Neuro-Psiquiatr., v. 63, n. 4, p. 1104-1112, 2005.

9. FRASER, M. Memory clinics and memory training. In: ARIE ,T. (Ed.). Recent advances in psychogeriatrics. London: Churchill Livingstone, 1992. p.105-115.

10. GRAFF, M. J. L.; VERNOOIJ-DASSEN, M. J. M.; THIJSSEN, M.; DEKKER, J.; HOEFNAGELS, W. H. L.; RIKKERT, M. G. M. O. Community based occupational therapy for patients with dementia and their care givers: randomised controlled trial. BMJ. v. 333, n. 7580, p. 1196- 196, 2006.

11. HERRERA, E.; CARAMELLI, P.; NITRINI, R. Estudo epidemiológico populacional de demência na cidade de Catanduva, Estado de São Paulo, Brasil. Rev. Psiquatr. Clin. v. 25 , p. $70-73,1998$.

12. LAW, M.; CARdoso, A. A.; MAGAlhãeS, L. V.; , 
MAGAlHÃES, <. C. (Org. Trad.). Medida Canadense de Desempenho Ocupacional (COPM). Belo Horizonte: Editora UFMG, 2009.

13. MACHADO, J. C. Doença de Alzheimer. In: FREITAS, E. V.; et al. Tratado de geriatria e gerontologia. Rio de Janeiro: Guanabara Koogan; 2006: 261-279.

14. NITRINI, R.; et al. Diagnóstico de doença de Alzheimer no Brasil: avaliação cognitiva e funcional. Recomendações do Departamento Científico de Neurologia Cognitiva e do Envelhecimento da Academia Brasileira de Neurologia. Arq Neuro-Psiquiatr., v. 63, n. 3a, p. 720-727, 2005.

15. NITRINI, R. Há sentido em utilizar o tratamento atualmente disponível para a doença de Alzheimer? Rev. Psiquiatr. Clín., v. 33 , n. 4, p. 214-217, 2006.

16. PFEFFER, R. I.; KUROSAKI, T. T.; HARRAH, C. H.; CHANCE, J. M.; FILIS, S. Measurement of functional activities in older adults in the community. J Gerontol., v. 37, p. $323-329,1982$.

17. TOGLIA, J. P. Treinamento e reabilitação cognitivoperceptual. In: NEISTADT, M. E.; CREPEAU, E. B. Willard \& Spackman - terapia ocupacional. $9^{\text {a }}$ ed. Rio de Janeiro: Guanabara Koogan, 2002. p. 298-418.

18. WINBLAD, B.; et al. Donepezil in patients with severe Alzheimer's disease: double-blind, parallel-group, placebocontrolled study. Lancet, v. 367, p. 1057-1065, 2006.

19. HOMMA, A.; et al. Long-term safety and efficacy of donepezil in patients with severe Alzheimer's Ddisease: results from a 52-week, open-label, multicenter, extension study in Japan. Dementia Geriatric Cogn Disord., v. 27, n. 3, p. 232239,2009

20. HOGAN, D. B.; et al. Diagnosis and treatment of dementia: 5. Nonpharmacologic and pharmacologic therapy for mild to moderate dementia. CMAJ. v. 179 , n. 10 , p. $1019-1026$, 2008 .

Recebido para publicação: 15/05/2011

Aceito para publicação: 20/06;2011 